University of Nebraska - Lincoln

DigitalCommons@University of Nebraska - Lincoln

$3-2008$

\title{
Use of a geometric rule or absolute vectors: Landmark use by Clark's nutcrackers (Nucifraga columbiana)
}

Debbie M. Kelly

University of Saskatchewan, Debbie.Kelly@usask.ca

Sarah Kippenbrock

University of Nebraska - Lincoln

Jennifer J. Templeton

Knox College, jtemplet@knox.edu

Alan Kamil

University of Nebraska - Lincoln, akamil1@unl.edu

Follow this and additional works at: https://digitalcommons.unl.edu/bioscibehavior

Part of the Behavior and Ethology Commons

Kelly, Debbie M.; Kippenbrock, Sarah; Templeton, Jennifer J.; and Kamil, Alan, "Use of a geometric rule or absolute vectors: Landmark use by Clark's nutcrackers (Nucifraga columbiana)" (2008). Papers in Behavior and Biological Sciences. 41.

https://digitalcommons.unl.edu/bioscibehavior/41

This Article is brought to you for free and open access by the Papers in the Biological Sciences at DigitalCommons@University of Nebraska - Lincoln. It has been accepted for inclusion in Papers in Behavior and Biological Sciences by an authorized administrator of DigitalCommons@University of Nebraska - Lincoln. 
Published in Brain Research Bulletin 76 (2008), pp. 293-299; doi: 10.1016/j.brainresbull.2008.02.008

Copyright $(2008$ Elsevier Inc. Used by permission.

Submitted September 7, 2007; revised December 9, 2007; accepted February 6, 2008; published online March 3, 2008.

RESEARCH REPORT

\title{
Use of a geometric rule or absolute vectors: Landmark use by Clark's nutcrackers (Nucifraga columbiana)
}

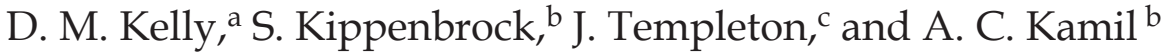 \\ a University of Saskatchewan, 9 Campus Drive, Department of Psychology, Saskatoon, Saskatchewan, S7N 5A5 Canada \\ ${ }^{\mathrm{b}}$ Department of Biological Sciences, University of Nebraska-Lincoln Lincoln, NE, USA \\ ${ }^{\mathrm{c}}$ Department of Biological Sciences, Knox College, Glaesburg, IL, USA \\ Corresponding author - D. M. Kelly, tel 306 966-2941, fax 306 966-6630, email Debbie.Kelly@usask.ca
}

\begin{abstract}
Clark's nutcrackers (Nucifraga columbiana) were trained to search for a hidden goal located in the center of a four-landmark array. Upon completion of training, the nutcrackers were presented with tests that expanded the landmark array in the east-west direction, north-south direction and in both directions simultaneously. Although the birds learned to search accurately at the center of the landmark array during training, this search pattern did not transfer to the expansion tests. The nutcrackers searched at locations defined by absolute distance and/or direction relationships with landmarks in the training array. These results contrast with those from experiments with nutcrackers in which an abstract geometric rule was learned. This difference appears due to differences in the experimental paradigms used during training.
\end{abstract}

Keywords: Clark's nutcracker, geometry, landmarks, absolute and relative metrics

Many animals use landmarks to accurately return to a previously visited site. This navigational strategy requires that an individual encode relevant spatial information about stable objects in the environment, retain this information for a period of time and subsequently retrieve the necessary details to guide a successful return. Studies of landmark use have shown that many species use landmarks to relocate target sites (e.g. bees, gerbils, pigeons, and nutcrackers) [1, 5, 2, 3, 19, respectively]. The landmark properties which are encoded, however, may differ markedly across species and situations [17, 18].

The transformational approach is often used to examine landmark-based search behavior [15,4]. In this approach, an individual is trained to locate a hidden goal at a fixed distance and direction from an object or set of objects. Once the animal is accurately locating the goal, the arrangement of the landmarks is systematically manipulated. Which landmarks and landmark properties were initially encoded can then be determined by examining how the search pattern is affected by the experimental manipulations.

The transformational approach has been adopted to examine whether birds can use geometric relationships among landmarks [e.g., 6, 12, 13, 11]. Spetch et al. trained pigeons and humans to locate a hidden goal that was centered between four identical landmarks [13]. Once the participants were accurately locating the hidden goal, the researchers manipulated the landmark configuration by either expanding the array in the left-right dimension, making a rectangular array, or along the diagonal, making a larger square array than used in training. Whereas human participants continued to search in the middle of the modified arrays, pigeons searched at a location preserving the absolute landmark-goal distance and direction relationships to individual landmarks in the transformed array. It appeared that while humans were able to use a geometric rule, the pigeons encoded a more specific rule based on absolute distance and/or direction.

Research with chicks has also adopted a transformational approach to examine whether spatial relationships between landmarks and a goal location is encoded using absolute or relative metrics. Unlike Spetch et al., Tommasi and Vallortigara trained chicks to use the overall shape of an enclosed environment rather than an array of discrete landmarks [16]. This was accomplished by training two groups of chicks to search 
for food hidden in the center of a fully enclosed square-shaped environment. The environment was constructed such that all the interior walls of the apparatus were identical requiring the chicks to use the geometric properties of the room to locate the hidden food. For the group tested with a larger or expanded environment the chicks divided their choices between the center of the arena and a location defined by an absolute vector. The group of chicks tested with a smaller or contracted environment from training concentrated their search to the middle of the arena only. This result is difficult to interpret, however, because the search pattern was rather diffuse. In addition, had the chicks encoded the goal location using an absolute vector they would have been expected to search at a location outside of the small testing arena but that was not possible.

Taken together these results suggest that, at least under many conditions, birds will encode the absolute metric properties of the relationship between a goal and an environment or landmark array. Perhaps it is not surprising that the birds in the studies reviewed above used an absolute vector strategy to search for the goal given the training paradigms they experienced. These birds were provided with a single exemplar of a fixed goal-landmark relationship, in contrast to multiple exemplar training which has been shown to be important for relational encoding [21]. Single exemplar training may have encouraged the birds to encode one or more goal-landmark vector(s) rather than a more relational geometric rule. During testing, both bird species were presented with an expanded version of the training paradigm. Expanding the landmark array or the size of the apparatus alters the geometric relationship among landmarks or walls. Therefore, even if the birds had represented the landmark-landmark geometry this strategy might not be adopted when the distance among the landmarks is increased. Thus, although neither pigeons nor chicks showed primary use of a geometric rule in the studies discussed above, this may not be due to an inability to learn geometric relationships among landmarks, but rather a consequence of the specific training and testing paradigms employed.

Kamil and Jones used a novel approach to examine this issue $[9,10]$. They varied the relationship between a goal and the surrounding landmarks so that the goal location was defined by the geometric relationship between landmarks. In their studies, Clark's nutcrackers were trained to find a buried target located halfway between two landmarks. Unlike previous studies on geometric learning, the researchers trained the birds with multiple exemplars of the geometric relationship by using five inter-landmark distances. The nutcrackers learned the geometric rule rapidly, and transferred this rule use to both interpolated and extrapolated novel inter-landmark distances.

Several factors may account for the differing results with pigeons and chicks than that reported with nutcrackers. One such factor is the methodological differences in the training paradigms used. Perhaps training the nutcrackers with several inter-landmark distances encouraged the birds to solve the problem using a single geometric rule rather than multiple absolute vectors for each inter-landmark distance. Given that both the chicks and pigeons were trained with a single landmark configuration, this methodological difference may be the plausible explanation of the results.

Results reported from two recent studies support a methodological explanation. Similar to Kamil and Jones, Spetch et al. trained pigeons to locate a hidden goal, its position defined by the geometric configuration of two landmarks [14]. For one group of pigeons the goal was always west of the two landmarks and at a constant distance from each landmark. For the second group of pigeons the goal was again always to the west of the landmarks but was at a constant bearing $\left(45^{\circ}\right)$. The pigeons in both groups were able to locate the goal position and showed modest transfer to novel inter-landmark distances. These results show that when pigeons are provided with multiple training exemplars they may indeed be able to use a geometric rule similar to that reported for nutcrackers, albeit with less accuracy.

The current experiment was designed to test nutcrackers with a paradigm similar to that of Spetch et al., which encouraged pigeons to adopt an absolute goal-landmark-encoding strategy [13]. On the one hand, if the nutcrackers learn a geometric rule with exposure to a single training exemplar this would suggest a species difference between pigeons and nutcrackers in the flexibility to learn a geometric relationship or rule. On the other hand, if the nutcrackers respond in a manner similar to pigeons and encode an absolute spatial relationship, we can be more confident in concluding that the differential use of spatial relationships in landmark arrays are more likely due to the methodology used rather than a quantitative species difference in the ability to use abstract geometric relationships.

\section{Methods}

\section{1. Subjects}

Five wild-caught adult Clark's nutcrackers (Nucifraga columbiana) served as subjects. All birds had previous experience with unrelated experiments but were naïve to spatial search tasks. The birds were maintained at $90 \%$ of their free feeding weight with pine seeds obtained during experimental sessions and supplemental feedings consisting of turkey starter, sunflower seeds, parrot pellets, mealworms, pine seeds, and vitamin supplement. They were housed individually in large cages with free access to water and grit. The colony was on a 14: 10 light-dark cycle with lights on at 6 a.m. The housing room was maintained at $22^{\circ} \mathrm{C}$.

\subsection{Apparatus}

The experiment was conducted in a $4.4 \mathrm{~m} \times 2.7 \mathrm{~m}$ room with a smoked glass window for observation and an entrance door located along the east wall. A porthole, allowing the birds to enter and exit the experimental room, was located directly below the observation window. A holding cage for the birds was located flush against the porthole outside the experimental room. The testing arena was a wooden floor raised $7 \mathrm{~cm}$ above the concrete floor of the room. A $120 \mathrm{~cm} \times 120 \mathrm{~cm}$ grid, with $10 \mathrm{~cm}$ squares was marked on the wooden floor. The origin of the grid was centered in the room dividing it into four equal quadrants. Approximately $2 \mathrm{~cm}$ of aspen chip substrate covered the entire surface of the floor.

A Panasonic WV-BL200 black and white video camera was mounted in the center of the experimental room. Connected to the camera was a Sony GV-D300 NTSC digital videocassette recorder and a Panasonic TR-930 video monitor. This configuration allowed for monitoring and recording of all experimental sessions.

Four uniquely painted landmarks were constructed from polyvinyl chloride (pvc) pipe, each landmark measuring $60 \mathrm{~cm}$ tall with a $2.5-\mathrm{cm}$ diameter. The four color patterns were solid yellow, solid green, black with three yellow stripes (every $5 \mathrm{~cm}$ starting from the top), and white with five red stripes (every $3 \mathrm{~cm}$ starting from the top). For ease of reference the last two landmarks will be referred to as the black landmark and the white landmark, respectively. 


\subsection{General procedures}

Birds were carried from the colony room to the experimental room and placed in the holding cage. Prior to the start of each trial, the landmarks (with the exception of habituation trials in which no landmarks were present) and goal were positioned according to a predetermined schedule. The goal locations were randomly chosen from all possible goal positions without replacement. To begin each trial, the lights in the holding area were turned off while the lights in the experimental room were illuminated. The sliding door covering the porthole was opened to allow the bird access to the experimental room. All trials were videotaped starting just prior to opening the porthole door. Each trial lasted until the bird found all the seeds (see below) or until 40 probes were made in the substrate (with the exception of the test trials which ended after 7 probes). After completion of the trial, the lights in the experimental room were extinguished, the lights in the holding area were illuminated and the video recording was stopped. Once the bird returned to the holding cage the door to the porthole was closed securing the bird in the holding cage until the beginning of the next trial. Upon completion of each daily session the bird was returned to its individual cage in the colony room.

\subsection{Habituation}

Prior to the beginning of training the birds were given five habituation sessions, one trial per session, to familiarize them with the experimental room. Two pine seeds, with the shells removed, were placed on a lid from a 35-mm film container on the surface of the substrate. The position of the lid was randomly determined for each session. No landmarks were present during these trials. Each trial lasted until the bird ate the available pine seeds or until approximately $20 \mathrm{~min}$ elapsed, whichever occurred first.

\subsection{Training}

The four landmarks were placed in a square array measuring $50 \mathrm{~cm} \times$ $50 \mathrm{~cm}$. The order of landmarks, clockwise from the northwest corner, was white, green, yellow, and black. For each training trial the location of the goal, and thus the landmark array, was randomly chosen from a possible 92 goal locations, 23 locations per quadrant. This allowed each possible goal location to be separated by $20 \mathrm{~cm}$ along the $x$-axis and 10 $\mathrm{cm}$ along the $y$-axis. The only restriction on the selection of the goal location for each trial was that it had to appear in three of the four quadrants of the grid (see Section 1.2) once per session. The relationship between the landmarks and the goal remained stable across trials.

Four stages were conducted to train the nutcrackers to accurately locate the position of the hidden goal. For the first stage of training, the initial two trials of the day were conducted with the goal showing. Only the third and final trial was conducted with the seeds completely buried. This stage lasted 10 days. For the second stage, the target was showing on only the first trial; the last two trials had the seeds completely buried. This training stage lasted 4 days. During the third stage, the seeds were buried on all three trials. This stage lasted 4 days. In the final stage of training, one of the trials was a non-reinforced trial. Non-reinforced trials were conducted to familiarize the birds in the absence of reinforcement because all testing trials were conducted without reinforcement.

\subsection{Testing}

Testing immediately followed completion of training and was conducted in two consecutive stages. Each testing session consisted of four trials. Three of these four trials were reinforced trials identical to the final stage of training (i.e., the goal was completely buried). The remaining trial was a non-reinforced test trial. Because no goal was present on this test trial, the non-reinforced test trial was ended, by turning out the lights in the room, after the bird made seven probes in the substrate. In the first phase of testing, four types of testing conditions were given. The landmark array in control tests was identical to that in final training trials except no reinforcement was available. The three remaining test types manipulated the arrangement of the four-landmark array. On east-west (E-W) expansion tests, the array of land- marks were expanded to $65 \mathrm{~cm}$ in the east-west direction only. Thus, the shape of the landmark array was distorted from a square configuration to a rectangular configuration with the long axis in the E-W direction. On north-south (N-S) expansion tests, the array of landmarks was expanded to $65 \mathrm{~cm}$ in the north-south direction only. Thus, the shape of the landmark array was once again distorted from a square configuration to a rectangular configuration but now the long axis was in the N-S direction. On Full expansion tests, the landmark array was expanded to $65 \mathrm{~cm}$ in both directions maintaining the square shape of the landmark array but increasing all of the landmark-landmark and landmark-goal distances. Similar to training, the landmark array was presented in different, randomly selected, locations within the room. The only restriction on the location of the goal was that it must appear in each of the grid's quadrants once per session. Each bird experienced a total of four blocks of testing (16 days) with each of the four testing conditions occurring once per block.

The second phase of testing followed immediately after the first testing phase and was identical to the first, but only two conditions were tested: a Control condition (identical to that used in the first phase) and a larger Full expansion test. On Full expansion tests, the distance between each landmark in the array was expanded to $100 \mathrm{~cm}$ in both the N-S and E-W directions. This manipulation maintained the square shape of the landmark array, but doubled the inter-landmark distance in comparison to the training array. Each bird received a total of six blocks of testing (12 days) with each of the two test types occurring once per block.

\subsection{Data recording and analysis}

In all stages of training and testing, buried seed trials and non-reinforced trials were videotaped. The videotaped trials were converted to digital movie files. For every trial, a separate static bitmap was created for the first five probes made by each nutcracker and the location of the landmark-goal array. Each bitmap was then scored to obtain the $x$ and $y$ coordinates for the five probes. The five coordinates were used to calculate three values for each probe: (a) east-west axis error (E-W error): the distance in centimeters from each probe to the goal location along the $x$-axis, (b) north-south axis error (N-S error): the distance in centimeters from each probe to the goal location along the $y$-axis, and (c) total error: the absolute value of the distance between the goal and each probe was averaged separately in the E-W and N-S axes, and then the Pythagorean theorem was applied to these values to calculate the total distance error.

Repeated measures analyses of variance (ANOVAs) were administered to analyze the data. Subsequent Fisher's least significant difference (LSD), multiple-comparisons tests were used only after significant $F$ ratios. Initial analyses used total error to compare the testing conditions. Subsequent ANOVAs were conducted to examine error as a function of E-W and N-S axes (axis was a factor in such ANOVAs). All significance testing was conducted at $\alpha=.05$.

\section{Results}

\subsection{Testing phase 1}

Total error was analyzed using a repeated measure ANOVA. A significant main effect of test type, $F(3,12)=4.19, p<.05$ was found. A subsequent Fisher's LSD test showed that the error in the Control condition $(M=8.1 \mathrm{~cm}$; S.E. $=1.46 \mathrm{~cm})$ was significantly less than error in both the N-S expansion condition ( $M$ $=12.7 \mathrm{~cm}$; S.E. $=2.89 \mathrm{~cm})$ and the full expansion condition $(M=$ $13.6 \mathrm{~cm}$; S.E. $=2.44 \mathrm{~cm}$; see Figure 1). Although error was greater in the $\mathrm{E}-\mathrm{W}$ expansion test $(M=10.0 \mathrm{~cm}$; $\mathrm{S} . \mathrm{E} .=3.35 \mathrm{~cm})$ than in the Control condition, this difference was not significant.

To examine possible differences in search accuracy, total error was divided into E-W and N-S components, and separate analyses of each component carried out. No significant differences were found between the test types along the E-W 


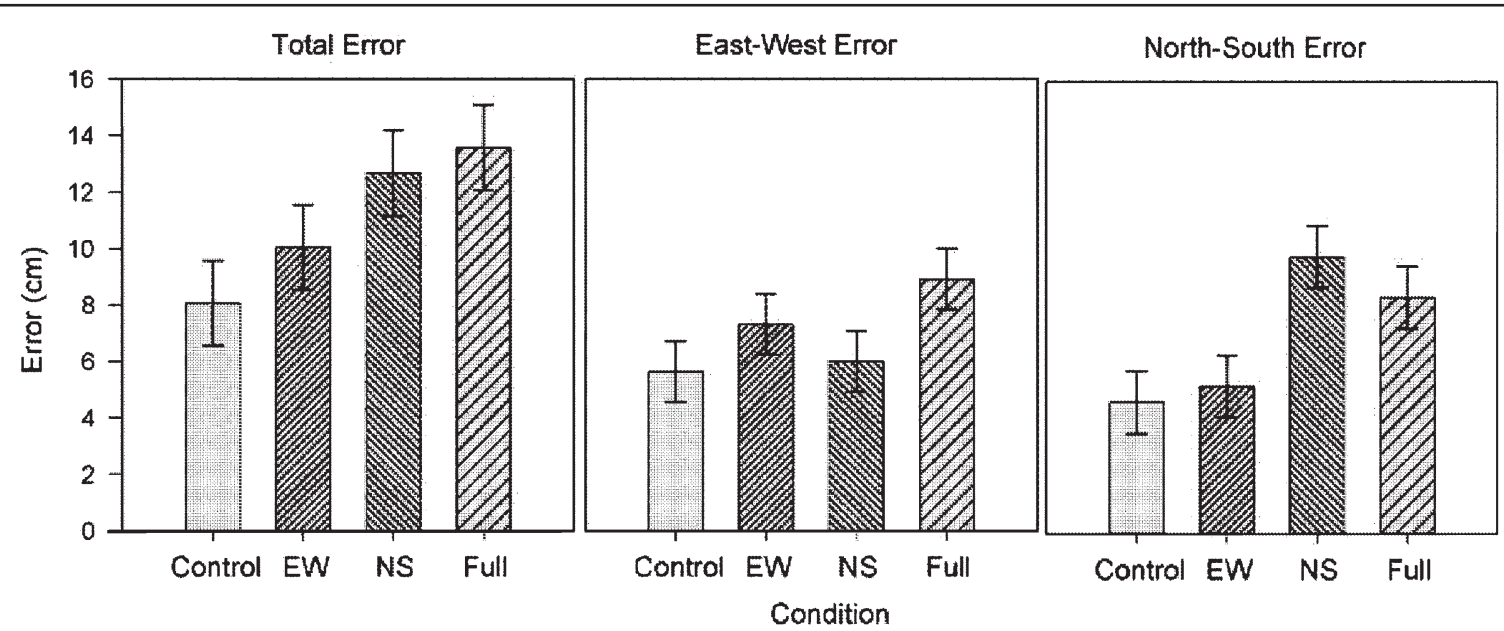

Figure 1. Testing phase 1: the amount of error, measured in centimeters, is shown for each of the four testing conditions. From left to right the panels show: total error, error along the east-west axis and error along the north-south axis, respectively.
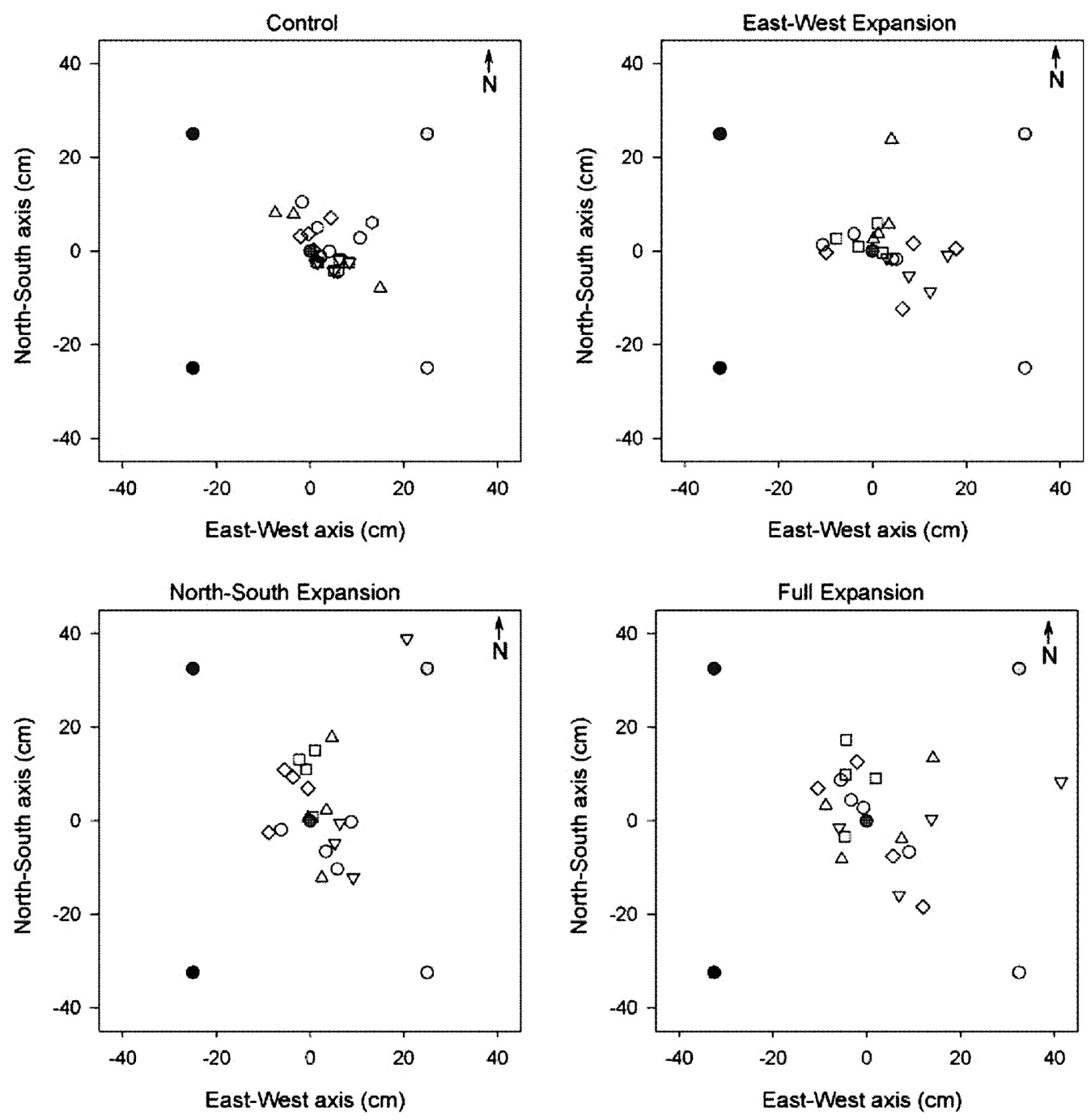

Figure 2. Testing phase 1: the location of the mean first five probes for each trial are represented. Each nutcracker is depicted by a different symbol. The panels from top left to bottom right depict the search locations for each of the four testing conditions: Control, East-West expansion, North-South expansion, and Full expansion, respectively. In each of the four panels, the five shaded circles represent the four landmarks and the location of the center of the array. Note that the white-colored landmark in the North-west position is shaded in this figure such that it stands apart from the probe symbols. 


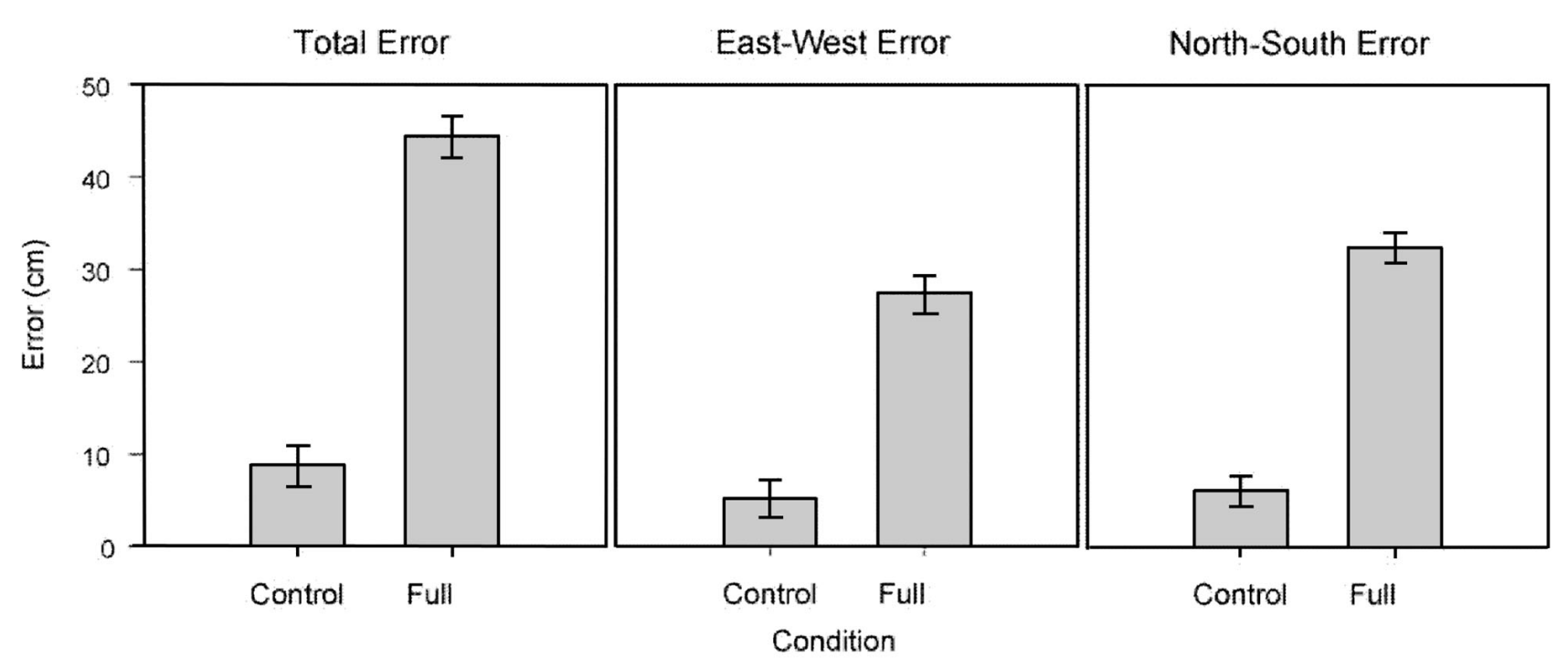

Figure 3. Testing Phase 2: the amount of error, measured in centimeters, is shown for the Control and Full Expansion testing conditions. From left to right the panels show: total error, error along the east-west axis, and error along the north-south axis, respectively.

axis $[F(3,12)=1.93, p>.05$; see Figure 1]. However, a significant main effect of test type was found for error in the N-S axis $[F(3,12)=5.04, p<.05]$. A subsequent Fisher's LSD test showed that error in the Control condition $(4.6 \mathrm{~cm})$ was significantly less than the error in the N-S expansion condition $(9.8 \mathrm{~cm})$ and in the full expansion condition $(8.4 \mathrm{~cm})$. Furthermore, error in the E-W expansion condition $(5.2 \mathrm{~cm})$ was significantly less than the error in the N-S expansion condition (see Figure 1).

The above analyses show that the birds' search patterns on the expansion tests differed significantly from the Control condition. This result suggests that the nutcrackers may not have been using a geometric rule to guide searching during the expansion tests. If the birds were using an absolute vector from a landmark or several landmarks to the goal then expanding the landmark array along one axis should increase the distribution of searches along the axis of expansion; there should be more variability along the expanded axis. For instance, in our E-W expansion test we would predict that the birds should increase their search distribution more in the E-W axis than in the N-S axis and vice versa during the N-S expansion test. Expanding along both axes, as during the Full expansion test should show no differences in the variance of search distribution along the two axes.

To examine the hypothesis that variance should be greater along the axis of expansion we computed an $F$-test for equality of variances [7]. Because we were specifically interested in the variance of the search distribution we first examined our data for possible multivariate outliers. Three outliers were removed according to the Mahalanobis distance test $\left(T^{2}=8.53,31.83\right.$, 38.27; all $\left.p^{\prime} \mathrm{s} \leq .05\right)$. We found that the birds showed greater variance in search distribution along the $x$-axis in comparison to the $y$-axis in the E-W expansion test $\left(F_{0.05(19,19)}=2.941\right)$ and more variance along the $y$-axis in comparison to the $x$-axis in the N-S expansion test $\left(F_{0.01}(19,19)=3.310\right)$. There was no significant difference in variance along the $x$ - and $y$-axes during the control and Full expansion tests $\left(F_{0.05(20,20)}=1.245\right.$ and $F_{0.05}$ $(19,19)=1.596$, respectively; see Figure 2$)$.

\subsection{Testing Phase 2}

Prior to beginning Phase 2, one nutcracker became ill and was dropped from the experiment. Therefore, the following analyses are conducted with four subjects. A repeated measures ANOVA revealed that error during the Control condition $(M$ $=8.8 \mathrm{~cm}$; S.E. $=0.55 \mathrm{~cm}$ ) was significantly less than error during the Full expansion test $[M=44.4 \mathrm{~cm}$; S.E. $=3.09 \mathrm{~cm}$; see Figure 3, $F(1,3)=137.35, p<.01]$.

Again, to examine possible differences in search accuracy, we divided total error into E-W and N-S components, and separate analyses were conducted. Significant differences were found among the test types for error in the E-W axis $[F(1,3)=$ 63.59, $p<.01]$. This shows that search error was lower in the Control condition $(5.2 \mathrm{~cm})$ than in the Full expansion condition (27.3 cm; see Figure 3). A significant difference for test type was also found for error in the N-S axis $[F(1,3)=141.42, p<.01]$. Showing that search error was lower in the Control condition $(6.1 \mathrm{~cm})$ than in the Full expansion test $(32.5 \mathrm{~cm}$; see Figure 3$)$.

As in Phase 1, we compared variance in the E-W and N$S$ axes. In this phase of testing we expanded the landmarks proportionally along both axes so we did not predict a significant difference between variance along the two axes. We again removed outliers according to the Mahalanobis distance test (three outliers were removed, $T^{2}=12.22,13.03,13.51$; all $p^{\prime} \mathrm{s} \leq$ .05). We found that the variance for the two axes did not differ during either the Control Condition $\left(F_{0.05(24,24)}=1.152\right)$ or the Full expansion test $\left(\mathrm{F}_{0.05(21,21)}=1.793\right.$; see Figure 4).

Overall, the expansion tests show that the nutcrackers did not focus their search to the center of the landmark array during the expansion tests. Rather, the birds showed an increase in search distribution in the direction of the landmark expansion. This result shows that the birds had not encoded the four-landmark array using a geometric rule but rather learned the location of the goal relative to one or more of the individual landmarks. Thus, increasing the landmark-landmark or landmark-goal distance increased the directional variance of the birds' search distribution. 

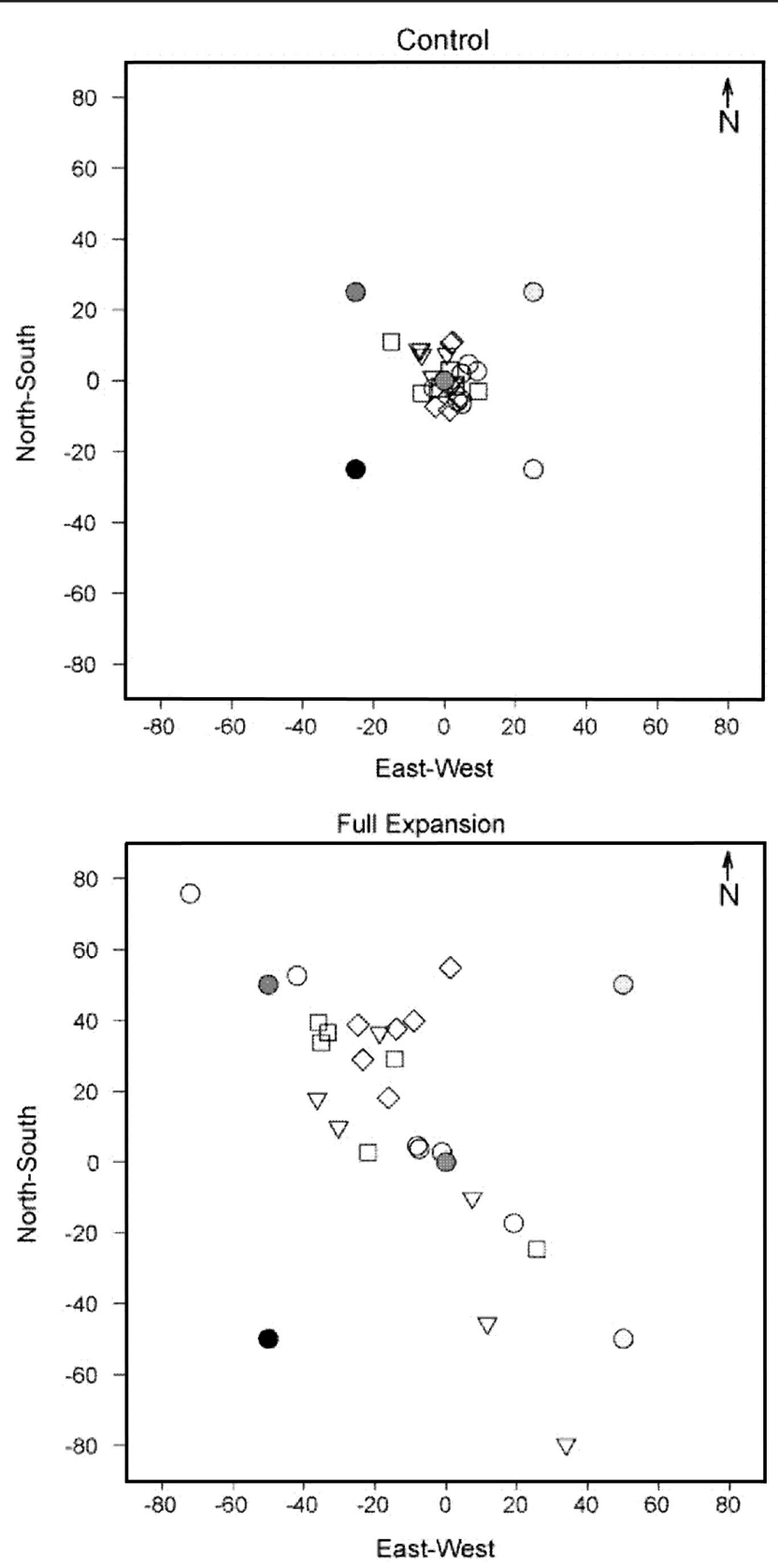

Figure 4. Testing Phase 2: the location of the mean first five digs for each trial are represented. Each nutcracker is depicted by a different symbol. The top panel shows search locations for the Control condition and the bottom panel shows search locations for the Full Expansion condition. In both panels the five shaded circles represent the four landmarks and the location of the center of the array. Note that the white-colored landmark in the North-west position is shaded in this figure such that it stands apart from the probe symbols.

\section{Discussion}

Nutcrackers learned to search for food hidden in the center of a four-landmark array. However, when the landmark array was expanded the nutcrackers no longer searched in the center of the array but rather distributed their search along the axis of expansion. Results from this study show that the nutcrackers were not using a geometric rule to encode the position of the hidden target but rather they searched for the goal location at a vector maintaining the training direction and distance from individual landmarks. In the first phase of testing, total error was greater in the expansion conditions than in the Control conditions (although in the E-W expansion this difference did not meet significance). Furthermore, when we examined the variance in search distribution we found support for our hypothesis that variance in search distribution should increase in the direction of the landmark expansion. This was shown by an increase in search variance along the $x$-axis in the E-W expansion test and along the $y$-axis in the N-S expansion test.

The results from this experiment, showing that the birds did not rely on a geometric strategy when searching on the expanded test trials, provide an important complement to the results reported by Kamil and Jones [9, 10]. Kamil and Jones showed that nutcrackers could learn to search for a hidden goal location using the geometric relationship between landmarks and goal position, transferring this geometric rule to novel inter-landmark distances. Although the nutcrackers in the current study readily learned to search in the center of the four-landmark array they did not transfer this search pattern when presented with the expansion tests. Instead, the search behavior of the nutcrackers was similar to that reported by Spetch et al. with pigeons [13]. Nutcrackers trained with a fixed, square array and then tested with an expanded array during probe tests searched at locations maintaining an absolute distance and/or direction from one or two landmarks, as did pigeons [13]. Thus it is most reasonable to conclude that the use of abstract geometric rules such as midpoint or halfway are strongly dependent on training procedures in which multiple exemplars are used. However, two additional points must be kept in mind.

First, there still appears to be quantitative differences between nutcrackers and pigeons in accuracy during these search tasks. This is in general agreement with the findings of Spetch et al. [14]. In this study, pigeons were trained with varying arrays using a protocol similar to that of Kamil and Jones [10]. The pigeons showed some evidence of geometric rule learning, but were much less accurate than nutcrackers. Similar results have also been reported by Jones et al. who used a comparative study to directly investigate the use of a geometric rule by pigeons and nutcrackers by examining the performance of both species on a very similar task (although conducted in two separate laboratories) [8]. Both species were trained to locate a hidden goal centered between two landmarks (similar to the half group in [10]). The nutcrackers and the pigeons were able to learn the task and successfully transfer to novel inter-landmark distances, although the search accuracy of pigeons was much lower than that of nutcrackers. The results from Jones et al. and Spetch et al. suggest that pigeons are capable of learning a geometric rule when trained with multiple exemplars of the training array.

Although pigeons and nutcrackers show use of a geometric rule, the accuracy with which the two species use this rule seems to differ. Spetch et al. reported that pigeons showed "modest transfer" to novel inter-landmark distances, whereas Kamil and Jones [10] report strong transfer by nutcrackers. Although a direct comparison cannot be made between the Spetch et al. study and the Kamil and Jones study, the results suggest that the nutcrackers showed more accurate transfer than the pigeons. Furthermore, in the Jones et al. study, where a more direct species comparison may be made, the nutcrack- 
ers again showed greater accuracy with novel inter-landmark distances when compared to the performance by the pigeons. This suggests that although methodological issues may account for some of the apparent differences in the ability of pigeons and nutcrackers to use a geometric rule $[13,10]$, interesting quantitative differences may exist between the two species.

Second, although pigeons and nutcrackers follow absolute metric rules when tested with array expansions following training with a single exemplar, results following training with multiple exemplars that maintained relative geometric relationships between goal and landmarks clearly demonstrate that these birds can learn such rules $[9,10,14,8]$. This raises the question of why they fail to demonstrate learning of relative rules following single exemplar training. The simplest explanation is that they do not learn relative geometric relationships when relative and absolute rules are both valid. It is also possible that they learn both absolute and relative rules when both are valid, but use absolute rules when there is a conflict between the two. One way to test this possibility would be to test for transfer between procedures. For example, would animals trained to the center of a square array with a single exemplar learn to respond accurately to multiple exemplars more or less rapidly than controls? If they only learned absolute rules during the original training, then negative transfer would be expected. But positive transfer would suggest that some more general rules had also been learned during training with a single exemplar. Studies of avian lateralization would likely not predict positive transfer given that many studies of spatial encoding show a right hemisphere dominance - relational encoding-even under binocular viewing [20]. The results from our present study suggest that incorporating multiple versus single exemplar training may prove to be an important methodological consideration for future studies of avian spatial learning and memory.

\section{Acknowledgments}

The experiments reported here were conducted by Sarah Kippenbrock and fulfilled partial requirement towards completion of her honors thesis. We thank Tim Suhr and Aleida J. Goodyear for their assistance in the conducting of the experiments. This research and manuscript preparation was supported by a National Institute of Mental Health MH61810 to Alan C. Kamil, and a Natural Sciences and Engineering Research Council of Canada postdoctoral fellowship and Discovery Grant to Debbie M. Kelly.

\section{References}

[1] B. A. Cartwright, T. S. Collett, How honeybees use landmarks to guide their return to a food source, Nature 295 (1982) 560-564.

[2] K. Cheng, Some psychophysics of the pigeon's use of landmarks, J. Comp. Physiol. A. 162 (1988) 815-826.

[3] K. Cheng, The vector sum model of pigeon landmark use, J. Exp. Psychol. Anim. Behav. Process. 15 (1989) 366-375.
[4] K. Cheng, M. L. Spetch, Mechanisms of landmark use in mammals and birds, in: S. Healy (Ed.), Spatial Representation in Animals, Oxford University Press, Oxford, 1998, pp. 1-18.

[5] T. S. Collett, B. A. Cartwright, B. A. Smith, Landmarks learning and visuospatial memories in gerbils, J. Comp. Physiol. A. 158 (1986) 835-851.

[6] E. R. Gray, M. L. Spetch, Pigeons encode absolute distance but relational direction from landmarks and walls, J. Exp. Psychol. Anim. Behav. Process. 32 (2006) 474-480.

[7] W. I. Hays, Statistics, 3rd ed. , Harcourt Brace College Publishers, Fort Worth, 1994.

[8] J. E. Jones, E. Antoniadis, S. J. Shettleworth, A. C. Kamil, A comparative study of geometric rule learning in nutcrackers (Nucifraga columbiana), pigeons (Columba livia), and jackdaws (Corvus monedula), J. Comp. Psychol. 116 (2002) 350-356.

[9] A. C. Kamil, J. E. Jones, The seed-storing corvid Clark's nutcracker learns geometric relationship among landmarks, Nature 390 (1997) 276-279.

[10] A. C. Kamil, J. E. Jones, Geometric rule learning by Clark's nutcrackers (Nucifraga columbiana), J. Exp. Psychol. , Anim. Behav. Process. 26 (2000) 439-453.

[11] D. M. Kelly, M. L. Spetch, Pigeons encode relative geometry, J. Exp. Psychol. Anim. Behav. Process. 27 (2001) 417-422.

[12] M. L. Spetch, K. Cheng, S. E. MacDonald, Learning the configuration of a landmark array. I. Touch screen studies with pigeons and humans, J. Comp. Psychol. 110 (1996) 55-68.

[13] M. L. Spetch, K. Cheng, S. E. MacDonald, B. Linkenhoker, D. M. Kelly, S. Doerkson, Use of landmark configuration by pigeons and humans. II. Generality across search tasks, J. Comp. Psychol. 111 (1997) 14-24.

[14] M. L. Spetch, T. B. Rust, A. C. Kamil, J. E. Jones, Searching by rules: pigeons' (Columba livia) landmark-based search according to constant bearing or constant distance, J. Comp. Psychol. 117 (2003) 123-132.

[15] N. Tinbergen, The Animal in its World, Harvard Press, Cambridge, 1972.

[16] L. Tommasi, G. Vallortigara, Searching for center: spatial cognition in the domestic chick (Gallus gallus), J. Exp. Psychol. Anim. Behav. Process. 26 (2000) 477-486.

[17] L. Tommasi, G. Vallortigara, Encoding geometric and landmark information in the left and right hemispheres of the avian brain, Behav. Neurosci. 115 (2001) 602-613.

[18] G. Vallortigara, P. Pagni, V. A. Sovrano, Separate geometric and nongeometric modules for spatial reorientation: evidence from a lopsided animal brain, J. Cognit. Neurosci. 16 (2004) 390-400.

[19] S. B. Vander Wall, An experimental analysis of cache recovery in Clark's nutcrackers, Anim. Behav. 30 (1982) 84-94.

[20] J. Vauclair, Y. Yamazaki, O. Güntürkün, The study of hemispheric specialization for categorical and coordinate spatial relations in animals, Neuropsychologia 44 (2006) 1524-1534.

[21] A. A. Wright, R. G. Cook, J. J. Rivera, S. F. Sands, J. D. Delius, Concept learning by pigeons: marching-to-sample with trial-unique video picture stimuli, Anim. Learn. Behav. 16 (1988) 436-444. 